\title{
Capacitación de recursos humanos en salud materna en Bolivia, Colombia, Ecuador, Guatemala, Haití, Honduras, Nicaragua y Perú: descripción de la experiencia*
}

\author{
Human Resource Training in Maternal Care in Bolivia, Colombia, Ecuador, Guatemala, Haiti, Honduras, \\ Nicaragua and Peru: An Experience Description
}

Treinamento de recursos humanos em saúde materna na Bolívia, Colômbia, Equador, Guatemala, Haiti, Honduras, Nicarágua e Peru: descrição da experiência

Silvia Helena De Bortoli Cassiani a 1.

cassianis@paho.org

DOI: https://doi.org/10.11144/Javeriana.ie21-2.crhs

Jovita Ortiz Contreras

Universidad de Chile, Chile

Fecha de recepción: 28 Octubre 2018

Amelia Brandt ${ }^{2}$.

Fecha de aprobación: 15 Abril 2019

Lorena Binfa Esbir

Universidad de Chile, Chile

Fernando A Menezes da Silva ${ }^{3 .}$

\section{Resumen:}

Introducción: América Latina y el Caribe han disminuido la mortalidad materna; sin embargo, aún poseen países con altas tasas, lo que refleja inequidades en el acceso a servicios de salud. La capacitación de recurso humano competente en atención primaria es una de las principales estrategias para abordar la problemática. Objetivo: describir la experiencia de planificación, implementación y evaluación de un programa de capacitaciones en salud materna en países de la región (Bolivia, Colombia, Ecuador, Guatemala, Honduras, Nicaragua, Haití y Perú). Metodología: entre 2016 y 2019, el Departamento de Sistemas y Servicios de Salud y la Unidad de Recursos Humanos de la Organización Panamericana de la Salud, junto a centros colaboradores, universidades y al Centro Latinoamericano de Perinatología y Salud de la Mujer, realizaron un levantamiento de necesidades de capacitación, desarrollaron plan de formación e implementaron capacitaciones para personal profesional y no profesional en salud materna. Resultados: las necesidades de capacitación detectadas fueron liderazgo en salud, cuidados prenatales, parto, posparto, emergencias obstétricas y planificación familiar. Estas se consideraron en capacitaciones presenciales y virtuales, con 71 entrenadores de 7 países, quienes a su vez las reprodujeron para más de 4000 personas. Las evaluaciones fueron muy positivas y se espera determinar el impacto en salud en futuros proyectos. Conclusiones: es fundamental la detección de necesidades y el trabajo colaborativo para realizar capacitaciones acordes en los países de la región, que permita contar con recurso humano motivado y competente para aumentar el acceso y la cobertura en la atención en salud materna de calidad.

Palabras clave: educación, educación continua, salud materna, recursos humanos.

\section{Abstract:}

Introduction: Latin America and the Caribbean have reduced the maternal mortality rates. However, some of these countries still have high rates, which indicates the inequity in the access to the health services. The human resource training competent in primary health care is one of the main strategies to approach this problem. Objective: To describe the experience of planning, implementing and evaluating a training program in maternal health in countries of this region (Bolivia, Colombia, Ecuador, Guatemala, Honduras, Nicaragua, Haiti and Peru). Methodology: Between 2016 and 2019, the Department of Health Systems

Notas de autor

1. Organización Panamericana de la Salud

2. Organización Panamericana de la Salud

3. Organización Panamericana de la Salud

a Autora de correspondencia. Correo electrónico: cassianis@paho.org 
and Services and the Human Resource Unit of the Pan-American Health Organization, together with some collaborative centers, universities and the Latin-American Center for Perinatology and Women's Health, carried out a survey on training needs, developed a formation plan and implemented the training among both professional and non-professional staff in relation to maternal health. Results: The training needs identified were Health Leadership, Prenatal Care, Labor, Postpartum Care, Obstetric Emergencies, and Family Planning. These needs are addressed both in the online and in-person training with 71 trainers from seven countries, who reproduced them for more than 4000 people. The evaluations were very positive and the health impact in future Project is yet to be determined. Conclusions: It is crucial to identify the needs and to do collaborative work in order to carry out the pertinent training in our countries. This would allow to count on the human talent, motivated and competent, in order to increase the access and coverage of high quality maternal health care.

Keywords: education, continuing education, maternal health, human resources.

\section{Resumo:}

Introdução: América Latina e Caribe diminuíram a mortalidade materna; no entanto, ainda têm países com altas taxas, refletindo iniquidades no acesso a serviços de saúde. O treinamento de recurso humano competente em atenção primária é uma das principais estratégias para abordar a problemática. Objetivo: Descrever a experiência de planejamento, implementação e avaliação de um programa de capacitações em saúde materna em países da região (Bolívia, Colômbia, Equador, Guatemala, Honduras, Nicarágua, Haiti e Peru). Metodologia: Entre 2016 e 2019, o Departamento de Sistemas e Serviços de Saúde e a Unidade de Recursos Humanos da Organização Pan-americana da Saúde, junto com centros colaboradores, universidades e o Centro Latino-americano de Perinatologia e Saúde da Mulher realizaram um inquérito de necessidades de treinamento, desenvolveram plano de formação e implementaram treinamentos para pessoal profissional e não profissional em saúde materna. Resultados: As necessidades de treino detectadas foram liderança em saúde, cuidados pré-natais, parto, pós-parto, emergências obstétricas e planejamento familiar. Estas foram consideradas em treinos presenciais e virtuais, com 71 treinadores de 7 países, quem por sua vez as reproduziram para mais de 4000 pessoas. As avaliações foram muito positivas e espera-se determinar o impacto em saúde em projetos futuros. Conclusões: É fundamental a detecção de necessidades e trabalho colaborativo para realizar treinamentos acordes nos países da região, que permitam contar com recurso humano motivado e competente para aumentar o acesso e a cobertura na atenção em saúde materna de qualidade.

Palavras-chave: educação, educação continua, saúde materna, recursos humanos.

\section{Introducción}

La Organización Panamericana de la Salud y la Organización Mundial de la Salud (OPS/OMS) consideran la atención primaria de salud (APS) un componente clave para lograr una salud sostenida y equitativa en las Américas. La evidencia ha mostrado que hay más probabilidades de que los sistemas de salud centrados consistentemente en la APS tengan mejores y más equitativos resultados en salud, mantengan los costos de salud más bajos, sean más eficientes y obtengan una mayor tasa de satisfacción que aquellos sistemas con un enfoque débil en la APS (1).

Se ha señalado que aumentar el acceso a la atención otorgada por personal calificado, en sistemas de salud basados en la APS, es una estrategia crítica para lograr la promoción de la salud y la prevención de enfermedades (2). Lo anterior también se ratificó en la Conferencia de Mundial sobre Atención Primaria de Astaná, donde se instó a los Estados a proveer:

[...] atención primaria de salud y servicios de salud de gran calidad, seguros, integrales, integrados, accesibles, disponibles y asequibles para todos y en todas partes, prestados con compasión, respeto y dignidad por profesionales de la salud bien formados, competentes, motivados y comprometidos. (3)

Sin embargo, las proyecciones mundiales actuales prevén una escasez de 12,9 millones de trabajadores de la salud para el año 2035. Adicionalmente, once países de la región tendrán un déficit de menos de 25 médicos, enfermeras y obstetras certificadas por cada 10.000 habitantes (4,5). Para abordar esta escasez, la OPS/OMS sugieren una mayor inversión en la capacitación de los trabajadores del área de la salud para producir un mayor número de profesionales competentes, motivados, bien distribuidos y reconocidos para brindar servicios de salud que satisfagan las necesidades de la población (6). 
La salud materna es un tema prioritario para la OPS/OMS, toda vez que las complicaciones evitables relacionadas con el embarazo, el parto y el puerperio causan la muerte a aproximadamente 830 mujeres por día en todo el mundo y que el 99\% de ellas ocurren países con menores ingresos (7). Ello produce en estos un mayor impacto en las expectativas de vida de la población (8), lo cual refleja las inequidades asociadas a la provisión de servicios de salud.

Si bien ha habido avances significativos en salud materna e infantil en la región de las Américas $(7,9)$, estos no han beneficiado por igual a todas las mujeres (10). Las muertes relacionadas con el embarazo son tres veces más para las madres en las comunidades indígenas, en comparación con las de la población general pues, a su vez, las gestantes con menos educación y con menos recursos económicos tienen mayores probabilidades de enfermar y morir $(10,11)$. Estas inequidades se pueden presentar dentro de una misma región, país e incluso zonas determinadas dentro de los mismos países (12).

Mejorar el acceso a una atención de calidad para gestantes y recién nacidos es un factor importante para reducir las muertes maternas e infantiles prevenibles (4,13). Así mismo, aumentar el número de profesionales de la salud capacitados en partería puede ayudar a disminuir la mortalidad y la morbilidad materna y neonatal, disminuir el número de intervenciones innecesarias y mejorar los resultados psicosociales y de salud pública (14).

En reconocimiento de este problema, el Departamento de Sistemas y Servicios de Salud/Unidad de Recursos Humanos para la Salud de la OPS/OMS desarrolló un proyecto de capacitación para mejorar las competencias de los trabajadores del área de la salud tanto profesionales como no profesionales que prestan atención en salud materna, que les permitiera otorgar una atención integrada con un enfoque equitativo e intercultural en la APS en América Latina y el Caribe. Este proyecto de capacitación formó parte de uno más amplio de Sistemas Integrados de Salud en Latinoamérica y el Caribe, que implementó la OPS/OMS en colaboración con fondos globales de Canadá para ocho países de la región de las Américas y del Caribe (Bolivia, Colombia, Ecuador, Guatemala, Haití, Honduras, Nicaragua y Perú).

Este artículo tiene como objetivo describir la experiencia de planificación, implementación y evaluación de un programa de capacitación en salud materna en los países participantes de la región de las Américas, para contribuir así en la socialización de estrategias de mejora de la salud de los niños y las madres en situaciones de vulnerabilidad en los ocho países mencionados.

\section{Metodología}

El proyecto se desarrolló en un periodo de cuatro años (2016-2019) y se organizó en etapas que dieran cuenta de la creación de los programas de capacitación para profesionales y para parteras tradicionales, trabajadores comunitarios y auxiliares de enfermería, así como su implementación en los países seleccionados. Las etapas I y II dan cuenta de aspectos metodológicos; la etapa III, de aspectos metodológicos y de resultados, y la etapa IV, de resultados propiamente. Dichas etapas se presentan de forma secuencial en la figura 1 y se describen a continuación. 


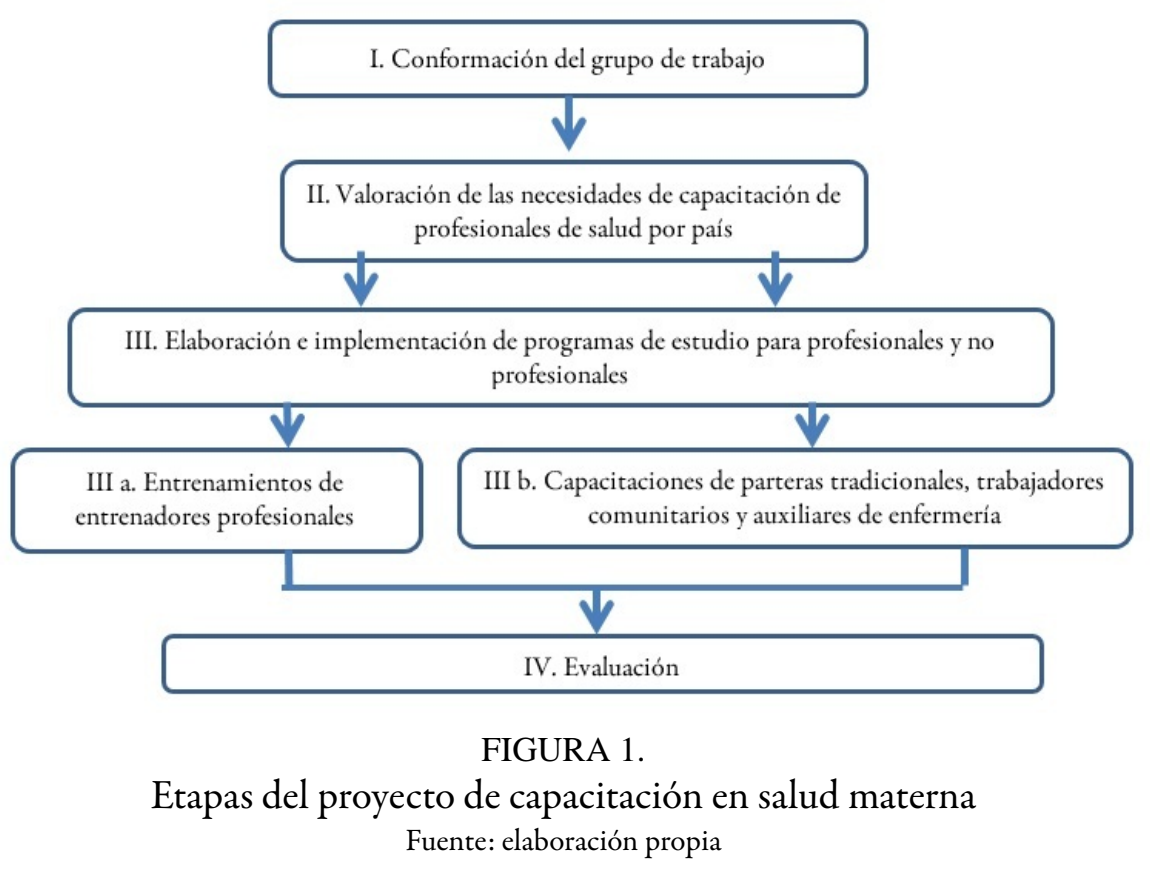

\section{Etapa 1: conformación del grupo de trabajo}

El Departamento de Sistemas y Servicios de Salud/Unidad de Recursos Humanos para la Salud de la OPS/ OMS convocó a trabajar en conjunto a distintas entidades y organismos como: las oficinas de la OPS/OMS en los países seleccionados, el Centro Latinoamericano de Perinatología y Salud de la Mujer (CLAP) y los centros colaboradores de la OPS/OMS de las universidades de Chile, Miami, Michigan, Pensilvania y São Paulo. El grupo de trabajo conformado analizó la programación e implementación del proyecto y se mantuvo en contacto a través de frecuentes reuniones virtuales.

\section{Etapa II: valoración de necesidades de capacitación por país}

Esta etapa consistió en la detección de las necesidades de formación en salud materna en todos los países participantes, con el propósito de que las capacitaciones se basaran en las reales necesidades de los países.

Para realizar esta etapa, en primer lugar, se elaboró una encuesta de detección de necesidades de formación en salud materna, la cual se centró en la identificación de los siguientes aspectos: 1) nivel educacional de parteras tradicionales, trabajadores de salud comunitarios, auxiliares de enfermería, enfermeras y obstetras; 2) identificación de las brechas en habilidades y determinación del conjunto de competencias básicas para cada grupo profesional; 3) roles y alcance de la práctica de los profesionales y cómo cada uno actualmente contribuye al sistema de salud del país; 4) regiones geográficas que se verían más beneficiadas de un programa de capacitación de educación continua; 5) prácticas culturales relacionadas con la salud materna, y 6) áreas temáticas y habilidades clínicas.

Adicionalmente, se realizó una revisión bibliográfica sobre salud materna en la región basada en el Cuestionario y el Manual de Análisis de Necesidades de Capacitación de Hennessy-Hicks (15) y la Guía de la OMS para la Evaluación Rápida de los Recursos Humanos para la Salud (16). Finalmente, el contenido de la encuesta fue revisado por representantes de la OPS/OMS en los países participantes y por expertos regionales en salud materna. 
En segundo lugar, se procedió a aplicar la encuesta previamente elaborada a informantes clave seleccionados por las oficinas locales de OPS/OMS. Posteriormente, el análisis se centró en sintetizar la información e identificar las necesidades por país y las necesidades generales de capacitación. Dichas necesidades dieron cuenta, sobre todo, de temáticas de liderazgo en salud, determinantes sociales y de temas específicos en salud materna, como cuidados prenatales, del parto, del posparto, emergencias obstétricas y planificación familiar, los cuales fueron considerados en la elaboración de las capacitaciones.

\section{Etapa III: elaboración e implementación de plan de capacitación para profesionales y no profesionales}

Para cumplir con las tareas de esta etapa, los centros colaboradores se encargaron de la elaboración y revisión de los contenidos de cada uno de los planes de estudio y, posteriormente, de la implementación de cada uno de ellos, a través de la formulación de las respectivas capacitaciones. Por último, se elaboraron dos planes diferenciados en términos de complejidad y formación: uno para profesionales de salud (enfermeras, enfermeras obstétricas, obstetras y médicos obstetras) y otro para capacitación de trabajadores comunitarios, parteras tradicionales y auxiliares de enfermería.

\section{Plan de capacitación para profesionales}

La elaboración del plan de capacitación para profesionales se realizó considerando su implementación a través de un formato semipresencial y se denominó formación de entrenador de entrenadores en salud materna. Para ello se consideró la incorporación del tema educación para adultos, además de las temáticas obtenidas de la valoración de necesidades. Esto, con el fin de permitir que la generación de competencias, así como el acceso a contenidos impartidos en el marco del proyecto, pudiera trascender su extensión y generara un efecto de escalamiento de los programas a través de una capacidad instalada de reproducción de estas. Los candidatos a entrenadores fueron seleccionados por la autoridad nacional de salud de cada país en colaboración con la oficina local de la OPS/OMS. Este programa, a su vez, se implementó en dos fases para facilitar el seguimiento y monitoreo de los países participantes.

Fase 1. Los países considerados en esta primera fase de entrenamiento de entrenadores en salud materna fueron: Honduras, Nicaragua, Colombia y Ecuador. La capacitación presencial de esta fase fue en la ciudad de Managua (Nicaragua), en agosto de 2017. En ella se impartió el primer módulo del plan de capacitación y se consideraron los temas de educación en adultos en salud materna, determinantes sociales, liderazgo en salud y violencia de género. Participaron 18 profesionales, quienes posteriormente continuaron la capacitación virtual a través del Campus Virtual de Salud Pública (CVSP) de la OMS/OPS.

Las metodologías de la capacitación presencial fueron netamente basadas en los principios de la educación de adultos $(17,18)$, participativas y consideraron en todo momento las experiencias de los participantes como eje de desarrollo y tratamiento de las temáticas. La evaluación de la experiencia y satisfacción de los profesionales fue muy positiva:

"Fue una experiencia muy enriquecedora, compartir con colegas de otros países y profundizar en las distintas metodologías para capacitar".

“... los temas y las formas en que se trataron fueron muy interesantes, me sirvió mucho para la aplicación en mi práctica profesional".

“... excelentes sesiones sincronizadas, le felicito fue una gran experiencia”.

El entrenamiento virtual de dicha fase, denominado Formación de entrenadorespara avanzar la competencia de recursos humanospara la salud materna en América Latina y el Caribe 2017, se llevó a cabo entre septiembre y diciembre de 2017 y consistió en cinco módulos virtuales con una asignación total de 126 horas. Las 
metodologías utilizadas en este contemplaron actividades de aprendizaje asincrónicas, como clases narradas en formato storyline y foros interactivos, las cuales la mayoría de los participantes consideraron útiles y de adecuada comprensión. Adicionalmente, se realizaron sesiones sincrónicas en formato WebEx en cada módulo, a fin de compartir conocimientos, reflexiones, experiencias y desafíos en torno a la temática; así como dos sesiones sincrónicas de VIH y a lactancia materna.

Como actividad final evaluada del curso, los participantes trabajaron en equipos nacionales para proporcionar un plan de capacitación de profesionales en salud materna, considerando los aspectos socioculturales de cada uno de los países. Respecto a la evaluación del curso por parte de los participantes, fue muy positiva. Algunos comentarios fueron:

"Considero que el curso fue muy dinámico, me sirvió para aclarar algunas dudas, refrescar conocimientos. Estar más comprometida con la salud de las mujeres durante el proceso reproductivo, para prevenir, modificar y tratar factores de riesgo que pueden llevar a la mujer y feto a estar en peligro su salud. Fue una experiencia muy rica”.

"El curso estuvo muy interesante y sobre todo los contenidos estaban bien enriquecedores, creo que va de acuerdo con la realidad de cada región y país".

Adicionalmente, las personas capacitadas como entrenadoras en la primera fase tuvieron la oportunidad de realizar el curso virtual Formación de Tutores para el Aprendizaje en Red. Edición 2018, Salud Materna, de 100 horas de duración, ofrecido el primer semestre del año 2018 por el CVSP.

Fase 2. En esta fase participaron 15 entrenadores seleccionados por los ministerios de Salud de Bolivia, Guatemala y Perú. Haití, por sus particularidades idiomáticas y políticas, fue considerado de forma paralela, lo cual se explica más adelante. El entrenamiento de entrenadores se llevó a cabo con la misma lógica de la primera fase: un entrenamiento presencial (módulo 1), realizado en Santa Cruz (Bolivia), en julio de 2018, y cinco módulos impartidos en el entrenamiento virtual. En la capacitación presencial se trataron las mismas temáticas de la primera fase, las metodologías fueron igualmente participativas y basadas en la educación para adultos. Las evaluaciones indicaron una experiencia positiva para los participantes y un aumento en el conocimiento de los temas de capacitación.

Para la capacitación virtual de esta fase se revisó y actualizó el material de la primera versión del curso virtual. Esta actualización estuvo a cargo del CLAP y del Centro Colaborador de la Universidad de Chile. Los cinco módulos revisados conformaron el Curso Virtual de Formación de Entrenadores para Avanzar la Competencia de Recursos Humanos para la Salud Materna en América Latina y el Caribe (2a edición, 2018), el cual se llevó a cabo entre agosto y noviembre de 2018. Adicionalmente, en el marco de colaboración de la OPS, se incorporaron al entrenamiento virtual siete profesionales del proyecto Hacia la salud universal de la población del Gran Chaco suramericano, pertenecientes a Argentina, Bolivia, Brasil y Paraguay.

El 91\#\% de las y los matriculados fueron aprobados en el curso. La evaluación de satisfacción del curso por parte de los participantes fue muy positiva, principalmente con relación a la pertinencia de los temas, al aprendizaje y a la importancia de compartir experiencias en el abordaje de la Salud Materna:

Comentarios de participantes del Curso Virtual, 2018:

El Curso me resultó muy productivo, satisfactorio porque tuve la oportunidad de una retroalimentación de los temas muy certeros que se han desarrollado en el curso. Es muy importante estar siempre actualizados con lo relacionado a la actividad o trabajo asumido, el cuidado de las personas es nuestra razón de ser.

El enfoque de los temas fue sumamente importante, nos dieron la oportunidad de compartir experiencias locales, las exigencias fueron también motivo de aprendizaje y si es muy útil para mejorar el trabajo en benéfico de la población materna.

Respecto a Haití, debido a la gran cantidad de organizaciones que trabajan en ese país, así como a la diversidad de planes de estudio de salud materna existentes, la colaboración se centró en el trabajo con el Ministerio de Salud. El primer paso fue la aplicación de la herramienta de necesidades, previamente traducida del inglés al francés por la OPS. El segundo paso fue la traducción del currículo de capacitación a nivel comunitario del español al francés. Este proceso se inició en noviembre de 2017 y finalizó en 2018. La implementación de la capacitación en Haití fue programada para marzo de 2019; sin embargo, debió ser 
suspendida por razones de contingencia nacional que fueron ajenas al proyecto. Independiente de la situación anterior, el material completo se encuentra traducido al francés para ser aplicado en cualquier contexto colaborativo.

\section{Plan de capacitación para no profesionales (parteras tradicionales, trabajadores comunitarios y auxiliares de enfermería}

Adicional a la elaboración e implementación de las capacitaciones orientadas a profesionales, se elaboró un plan de capacitación de parteras tradicionales, trabajadores comunitarios y auxiliares de enfermería. Este material fue organizado en seis módulos que contemplaron, al igual que el plan de capacitación de profesionales, los temas de liderazgo y determinantes sociales en salud, planificación familiar y VIH/ITS, educación prenatal, cuidados básicos en el parto, abordaje inicial de emergencias obstétricas y cuidados posparto.

El programa se plasmó en un Manual de consulta escrita, en un formato que consideró en cada módulo aspectos teóricos básicos de cada tema y actividades de reforzamiento y evaluación de lo aprendido en cada uno de ellos. Fue revisado por el grupo de trabajo, así como por los capacitadores que participaron en el programa Entrenador de Entrenadores y posteriormente fue traducido al inglés y francés.

La primera implementación de este programa se realizó en 2019, en el Amazonas de Perú, por ser una zona de alta mortalidad materna, con acceso geográfico limitado, donde la población es asistida en primera instancia por trabajadores comunitarios y por auxiliares de enfermería, muchos de ellos provenientes de poblaciones nativas.

Para facilitar la entrega de la información, representantes del equipo de trabajo y entrenadores nacionales realizaron, en primera instancia, una capacitación presencial de dos días, basada en el manual desarrollado en salud materna, dirigida a treinta obstetrices, quienes posteriormente estuvieron a cargo de repetir la capacitación a sus respectivos equipos de salud, considerando a los auxiliares y trabajadores comunitarios de sus respectivas zonas o microrredes (Chachapoyas, Candungos, Totora, Imaza, Tupac Amaru y Nivia).

Las metodologías utilizadas estuvieron orientadas a la reflexión y diálogo de saberes y a la adquisición de competencias de abordaje práctico inicial de emergencias obstétricas y de cuidados básicos en salud materna. Los participantes se mostraron muy satisfechos respecto a las metodologías y al abordaje de los temas y muy comprometidos con repetir la capacitación en sus respectivos lugares de trabajo:

"Muy contenta con la posibilidad de actualizar conocimientos en salud materna y tener la posibilidad de replicarlos...”. (Obstetriz Cap. Bagua, 2019)

"Me siento muy satisfecha de lo aprendido, necesitamos estar siempre actualizados en temas tan importantes para las mujeres y sus hijos". (Obstetriz Cap. Bagua, 2019)

Por su parte, las posteriores capacitaciones de las obstetrices estuvieron dirigidas a personal que asiste a población, principalmente de etnias nativas como ahuajun y wampis, quienes valoraron lo actualizado de los temas, así como la pertinencia cultural con la cual fueron abordadas. En la tabla 1 se puede observar el número de personas capacitadas por las obstetrices, según las zonas. 
TABLA 1.

Personas capacitadas según zonas en el Amazonas (Perú), 2019

\begin{tabular}{lrr}
\hline Zona & $\begin{array}{r}\text { Auxiliares o trabajadores } \\
\text { comunitarios }\end{array}$ & Profesionales \\
\hline Chachapoyas & 12 & 19 \\
\hline Candungos & 7 & 0 \\
\hline $\begin{array}{l}\text { Totora-Amazonas- } \\
\text { Perú }\end{array}$ & 12 & 10 \\
\hline Imaza & 20 & 0 \\
\hline Tupac Amaru & 2 & 1 \\
\hline Nivia & 20 & 0 \\
\hline Total & 73 & 30 \\
\hline
\end{tabular}

Fuente: elaboración propia

Otra forma de capacitar a personal no profesional fue la utilización de los contenidos trabajados por parte de los entrenadores nacionales, quienes en actividades propias de su rol local entregaron información a profesionales y auxiliares de enfermería en actividades de capacitación no necesariamente adscritas al proyecto, pero en las cuales utilizaron material emanado en el contexto de este (tabla 2).

TABLA 2.

Reporte de personas capacitadas por entrenadores nacionales posterior a su formación como tales en el marco del proyecto de capacitación en salud materna

\begin{tabular}{lr}
\hline País & $\begin{array}{r}\text { Número de personas } \\
\text { capacitadas en temáticas } \\
\text { de salud materna }\end{array}$ \\
\hline Colombia & 585 \\
\hline Ecuador & 765 \\
\hline Nicaragua & 1895 \\
\hline Bolivia & 356 \\
\hline Perú & 92 \\
\hline $\begin{array}{l}\text { Proyecto el } \\
\text { Chaco }\end{array}$ & 56 \\
\hline Total & 3749 \\
\hline
\end{tabular}

Fuente: elaboración propia

\section{Resultados}

\section{Etapa IV: evaluación de efectividad de los programas de capacitación en los países}

La evaluación del proyecto de capacitación, así como su efectividad, se demuestra principalmente considerando el número total de personas capacitadas en las diferentes instancias del curso (tabla 3). 
TABLA 3.

Número de personas capacitadas en salud materna

\begin{tabular}{lr}
\hline Instancia & $\begin{array}{r}\text { Número de } \\
\text { personas } \\
\text { capacitadas }\end{array}$ \\
\hline $\begin{array}{l}\text { Fase I y II: entrenadores } \\
\text { nacionales (cursos presenciales y } \\
\text { virtuales 2017 y 2018) }\end{array}$ & 41 \\
\hline $\begin{array}{l}\text { Reproducción de contenidos } \\
\text { informados }\end{array}$ & 3749 \\
\hline Curso virtual 2019 & 157 \\
\hline Curso presencial: Bagua 2019 & 30 \\
\hline $\begin{array}{l}\text { Reproducción de las } \\
\text { capacitaciones informadas (Perú) }\end{array}$ & 103 \\
\hline Total & 4080 \\
\hline
\end{tabular}

Fuente: elaboración propia

Adicionalmente, la efectividad pudo reflejarse mediante la generación de un tercer Curso Virtual para Avanzar en la Competencia de Recursos Humanos para la Salud Materna en América Latina. 2019, el cual permitió dar cuenta de dos resultados importantes: la demostración in vivo de las competencias de entrenadores nacionales y el aumento de la cobertura de entrega de información y capacitación que generó el proyecto.

Para el primer resultado logrado por dicho curso, entrenadores nacionales formados en las primeras etapas pudieron participar como tutores del curso, acompañados por profesoras del Centro Colaborador de la OPS/ OMS de la Universidad de Chile, donde cada uno estuvo a cargo de un grupo de aproximadamente 25 estudiantes, con la responsabilidad de guiar el proceso formativo de estos:

Mi experiencia individual fue maravillosa, agradezco la oportunidad que me dieron para apoyar como tutor virtual este curso... He tenido la oportunidad de pasar por los roles fundamentales para esta actividad, he sido estudiante y tutor virtual. (Entrenadora nacional, Bolivia)

No me queda más que decir que estoy feliz y muy agradecida de este bello curso... De ser parte de esta gran red de personas que buscan mejorar la Educación y la calidad de vida de las personas. Me he visto enormemente enriquecida, y estaré atenta a los próximos cursos. (Entrenadora nacional, Ecuador)

Por su parte, el curso propiamente dicho convocó a personal del área de la salud de los distintos países participantes, sobre todo servicios de APS, que trabajasen en áreas de salud materna. Así se logró una matrícula de 196 profesionales de Colombia, Ecuador, Honduras, Bolivia, Perú y Nicaragua. De los participantes, 157 fueron aprobados en el curso.

En cuanto a la satisfacción de los entrenadores nacionales al participar como tutoras, fue muy positiva con relación a la experiencia vivida y a la posibilidad de participar en un proyecto internacional y con contenidos tan actualizados.

Por su parte, los profesionales que cursaron el programa como estudiantes refirieron sentirse satisfechos en relación con la experiencia, aprendizaje y calidad de los contenidos:

Comentarios de participantes del Curso Virtual, 2019:

Un curso muy completo y oportuno, es muy importante tener estas actualizaciones para mejorar el conocimiento y la práctica del cuidado, implementar la promoción y prevención.

Me pareció un curso interesante, con información completa y accesible para la lectura, con una plataforma accesible, los foros de intercambios con muy buena información y opinión de mis compañeros.

Felicitaciones nuevamente a las docentes tutoras, realmente fue una experiencia excelente, gracias por enseñarnos a aprender y desaprender. 


\section{Discusión}

La atención otorgada por personal calificado y competente, con conocimiento actualizado, basada en la evidencia, centrada en las personas, segura y eficiente, es uno de los aspectos fundamentales para considerar en el marco de una atención de calidad (19). El propósito de este estudio fue compartir la experiencia en formación de recurso humano competente en Latinoamérica para el abordaje de la salud materna, teniendo cuenta todos los aspectos de calidad de atención, principalmente en APS. Como reflejo de ello, en el periodo de ejecución del proyecto, el cual fue básicamente de tres años, se capacitaron más de cuatro mil personas en las temáticas de salud materna en distintas instancias.

La satisfacción expresada por los profesionales que se formaron como entrenadores nacionales de los distintos países de Latinoamérica, así como por los profesionales que accedieron a las capacitaciones en salud materna, responde a una de las principales solicitudes emanadas de la Declaración de Astaná 2018: contar con profesionales de la salud bien formados, competentes, motivados y comprometidos para proveer atención digna a las personas (3). Las metodologías basadas en aprendizaje de adultos utilizadas tanto en la capación de profesionales y no profesionales como en las experiencias emanadas de cada una de las capacitaciones dieron cuenta de productos que forman parte de los recursos emanados del Proyecto de Capacitación en Salud Materna.

Por su parte, la motivación y el compromiso de los profesionales formados como entrenadores, así como de todas las personas que recibieron las capacitaciones, generó uno de los aspectos más valiosos emanados del Proyecto de Capacitación en Salud Materna: la capacidad formadora que dejó instalada en los países de América Latina y el Caribe. Esta capacidad se expresa en los siguientes productos:

1. Formación de 41 entrenadores nacionales en ocho países de Latinoamérica, quienes están certificados como tales.

2. Curso Virtual de Formación de Recurso Humano en Salud Materna creado con material actualizado y disponible en plataforma del CVSP, para ser clonado en nodos nacionales por las oficinas locales de la OPS.

3. Manual de capacitación en salud materna, el cual se encuentra disponible en español, inglés y francés.

4. Capacitación presencial en salud materna, disponible con programación, metodologías y contenido, para ser utilizada y repetida a solicitud.

5. Disposición de simuladores de atención en salud materna en tres países de Latinoamérica, con manual de uso en español e inglés, adquirido por medio del Programa PALTEX de la OPS/OMS.

Los cinco productos anteriores, la cantidad de personas entrenadas, el compromiso expresado por ellos y la incorporación de temáticas de liderazgo y de educación en adultos en los objetivos de las capacitaciones, los cuales son aspectos importantes en la generación de políticas de capacitación en salud (20), hacen prever una alta probabilidad de reproducción de los contenidos en distintos países de Latinoamérica, así como potencialmente a otros países a futuro, lo cual genera una capacidad inimaginable de acceso al conocimiento actualizado en la materia, pues disminuye brechas de acceso a la formación de muchos trabajadores de la salud.

El proyecto descrito ha utilizado las herramientas disponibles — de colaboración, recurso humano y tecnologías - que resultaron de la elaboración y disposición de un material de incalculable valor a disposición en Latinoamérica para ser utilizado tanto en forma presencial como virtual para contribuir en la disminución de brechas de formación y actualización en salud materna. Esto adquiere relevancia, ya que se ha demostrado que el contar con métodos presenciales y virtuales de educación en salud es una herramienta fundamental para la autogestión en salud y la disminución de dichas brechas (21).

Con el desarrollo de este proyecto se logró disponer de recurso humano competente y con conocimientos actualizados en la mejor evidencia disponible, además de un material sólido y múltiples estrategias metodológicas, lo cual, junto al advenimiento de recursos tecnológicos, principalmente el acceso cada vez más extendido de internet en la región de las Américas, hace de los productos de este proyecto una gran oportunidad de crecimiento y masificación de la entrega de información - que se había planteado como un desafío en los distintos países-, porque es potencialmente extensible el alcance de lo logrado. 
Finalmente, concluimos que el trabajo colaborativo entre distintas organizaciones, así como la disposición y uso de las tecnologías y la capacidad formadora instalada emanada de proyecto descrito, se traducen en un importante avance en la formación de recurso humano pertinente en salud materna en Latinoamérica, lo cual esperamos impacte positivamente en la salud materna e infantil en la región de las Américas.

\section{Agradecimientos}

Los autores agradecen a las siguientes personas: Helen Kwan, Rosina Cianelli, Kleyde Ventura, Flavia GomesSponholz, Juliana Cristina dos Santos Monteiro y Mark Cantrell y a todo el equipo del CVSP de la OPS/ OMS.

\section{Referencias}

1. PAHO/WHO. Renewing primary health care in the Americas: a position paper of the Pan American Health Organization/World Health Organization (PAHO/WHO) [Internet]. Washington; 2007 [citado 2019 Apr 21]. Disponible en: https://www.apps.who.int/medicinedocs/en/m/abstract/Js19055en/

2. PAHO/WHO. 52nd Directing Council: Resolution CD12.R12 human resources for health: Increasing access to qualified health workers in primary health care-based health systems [Internet]. Washington, DC; 2013 [citado 2019 Apr 21]. Disponible en: https://www.paho.org/hq/dmdocuments/2013/CD52-R13-e.pdf

3. OMS/UNICEF. Declaración de Astaná: Conferencia Mundial sobre Atención Primaria de Salud. Desde Alma-Ata hacia la cobertura sanitaria universal y los objetivos de Desarrollo sostenible [Internet]. Astaná, Kazajstán; 2018 [citado 2019 Jun 4]. Disponible en: https://www.who.int/docs/default-source/primary-health/declaration/gc phc-declaration-sp.pdf

4. WHO. WHO Global strategy on human resources for health: Workforce 2030 [Internet]. Genova: World Health Organization; 2017 [citado 2019 Apr 21]. Disponible en: https://www.who.int/hrh/resources/pub_globstrat hrh-2030/en/

5. PAHO/WHO. Strategy for Universal Access to Health and Universal Health Coverage [Internet]. Washington, DC; 2014 [citado 2019 Apr 21]. Disponible en: https://www.paho.org/uhexchange/index.php/en/uhexchange-documents/technical-information/26-stra tegy-for-universal-access-to-health-and-universal-health-coverage/file

6. Global Health Workforce Alliance and World Health Organization. WHO: A universal truth: no health without a workforce [Internet]. WHO. Genova: World Health Organization; 2014 [citado 2019 Apr 21]. Disponible en: https://www.who.int/workforcealliance/knowledge/resources/hrhreport2013/en/

7. OMS, UNICEF, UNFPA, Grupo del Banco Mundial y División de Población de las Naciones Unidas. OMS | Evolución de la mortalidad materna: 1990-2015 [Internet]. WHO. World Health Organization; 2016 [citado 2019 Apr 21]. Disponible en: https://www.who.int/reproductivehealth/publications/monitoring/maternal-m ortality-2015/es/

8. WHO. World health statistics overview 2019: monitoring health for the SDGs, sustainable development goals [Internet]. Geneva; 2019 [citado 2019 Jun 3]. Disponible en: https://www.apps.who.int/iris/bitstream/handle /10665/311696/WHO-DAD-2019.1-eng.pdf?sequence=1\&isAllowed=y

9. OPS. Indicadores básicos 2018: situación de Salud en las Américas [Internet]. Washington, DC: Organización Panamericana de la Salud; 2018 [citado 2019 Jun 4]. Disponible en: https://www.iris.paho.org/xmlui/handle $/ 123456789 / 49511$

10. The World Bank. Latin America: unequal access to health care is still no. 1 killer for moms and kids [Internet]. The World Bank. 2013 [citado 2019 Apr 21]. Disponible en: https://www.worldbank.org/en/news/feature/2013/ 09/11/mother-child-healthcare-inequalities-latin-america 
11. Tunçalp Ö, Souza J, Hindin M, Santos C, Oliveira T, Vogel J, et al. Education and severe maternal outcomes in developing countries: a multicountry cross-sectional survey. BJOG An Int J Obstet Gynaecol [Internet]. 2014 Mar [citado 2019 Jun 3];121:57-65. Disponible en: https://doi.wiley.com/10.1111/1471-0528.12634

12. Souza J, Tunçalp Ö, Vogel J, Bohren M, Widmer M, Oladapo O, et al. Obstetric transition: the pathway towards ending preventable maternal deaths. BJOG An Int J Obstet Gynaecol. 2014 Mar;121:1-4. https://doi.wiley.co $\mathrm{m} / 10.1111 / 1471-0528.12735$

13. World Health Organization, Human Reproduction Programme. Strategies toward ending preventable maternal mortality (EPMM) Executive Summary [Internet]. Genova; 2015 [citado 2018 Jun 4]. Disponible en: https://www.apps.who.int/iris/bitstream/handle/10665/153540/WHO_RHR_15.03_eng.pdf;jsessionid $=36 \mathrm{D} 2 \mathrm{FD} 32 \mathrm{E} 65 \mathrm{~F} 76 \mathrm{FA} 99 \mathrm{AEFF} 0999 \mathrm{CFAC} 29$ ? sequence $=1$

14. Renfrew MJ, McFadden A, Bastos MH, Campbell J, Channon AA, Cheung NF, et al. Midwifery and quality care: findings from a new evidence-informed framework for maternal and newborn care. Lancet [Internet]. $2014 \mathrm{Sep}$ 20 [citado 2019 Apr 21];384(9948):1129-45. Disponible en: https://www.ncbi.nlm.nih.gov/pubmed/24965 816

15. Hennessy D, Hicks C. Hennessy-hicks training needs analysis questionnaire and manual: For use at a local level to identify training and development needs [Internet]. 2011 [citado 2019 Apr 29]. Disponible en: https://www.w ho.int/workforcealliance/knowledge/toolkit/19.pdf?ua=1

16. WHO. A guide to rapid assessment of human resources for health. [Internet]. Geneva; 2004 [citado 2019 Apr 29]. Disponible en: https://www.who.int/hrh/tools/en/Rapid_Assessment_guide.pdf?ua=1

17. Taylor DCM, Hamdy H. Adult learning theories: Implications for learning and teaching in medical education: AMEE Guide No. 83. Med Teach [Internet]. 2013 Nov 4 [citado 2019 May 22];35(11):e1561-72. Disponible en: https://www.tandfonline.com/doi/full/10.3109/0142159X.2013.828153

18. Misch DA. Andragogy and medical education: are medical students internally motivated to learn? Adv Health Sci Educ Theory Pract [Internet]. 2002 [citado 2019 May 22];7(2):153-60. Disponible en: https://www.ncbi.nlm .nih.gov/pubmed/12075147

19. Tunçalp \#, Were W, MacLennan C, Oladapo O, Gülmezoglu A, Bahl R, et al. Quality of care for pregnant women and newborns-the WHO vision. BJOG An Int J Obstet Gynaecol. 2015;(122):1045-9. https://doi.wiley.com /10.1111/1471-0528.13451

20. Heiman HJ, Lerissa Smith L, McKool M, Mitchell DN, Bayer CR. Health policy training: A review of the literature. Int J Environ Res Public Health. 2016;13(1):20.

21. Lawn S, Zhi X, Morello A. An integrative review of e-learning in the delivery of self-management support training for health professionals. BMC Med Educ. 2017 Oct 10;17(1):183.

\section{Notas}

* Artículo de investigación

1. Conflictos de interés: los autores declaran no tener conflictos de interés.

\section{Licencia Creative Commons CC BY 4.0}

Cómo citar este artículo: De Bortoli Cassiani SH, Ortiz Contreras J, Brandt A, Binfa Esbir L, Menezes Da Silva FA. Capacitación de recursos humanos en salud materna en Bolivia, Colombia, Ecuador, Guatemala, Haití, Honduras, Nicaragua y Perú: descripción de la experiencia. Investig Enferm Imagen Desarr. 2020;21(2). https://doi.org/10.11144/Javeriana.ie21-2.crhs 